\title{
The Antibacterial Activity of a Fraction Extracted from Camel Urine against Mycobacterium tuberculosis Isolated from Tuberculosis Patients in Jeddah City
}

\author{
Raniya Sami Bakhsh ${ }^{1,2}$, Samah Omar Noor ${ }^{1,4}$, Faten Abdul Rahman Khorshid', Faten Ahmed \\ Alsulaimany'1, Abdulwahab Alzahrani' ${ }^{3}$, Azhar Abdullah Najjar'1, Ahmed M.M. Al-Hejin 1,4 \\ ${ }^{1}$ Department of Biological Sciences, Faculty of Science, P.O. Box 80203, King Abdulaziz University, Jeddah, 21589, Saudi Arabia. \\ ${ }^{2}$ MLS Department, Fakeeh College for Medical Sciences, Jeddah city, Saudi Arabia. \\ ${ }^{3}$ Molecular biology laboratory at King Fahd Armed Forces Hospital Jeddah city, Saudi Arabia \\ ${ }^{4}$ Microbiology Unit, King Fahd Medical Research Center, King Abdulaziz University, Jeddah, Saudi Arabia.
}

Correspondence Author: Samah Omar Noor, Department of Biological Sciences, Faculty of Science, P.0. Box 80203, King Abdulaziz University, Jeddah, 21589, Saudi Arabia.

Microbiology Unit, King Fahd Medical Research Center, King Abdulaziz University, Jeddah, Saudi Arabia.

Email: sonoor@kau.edu.sa

Received date: 12 February 2018, Accepted date: 12 April 2019, Online date: 20 April 2019

Copyright: (C) 2019 Raniya Sami Bakhsh et al., This is an open-access article distributed under the terms of the Creative Commons Attribution License, which permits unrestricted use, distribution, and reproduction in any medium, provided the original author and source are cre dited.

\begin{abstract}
This study aimed to investigate the effect of PMF (Prophet Medicine fraction) an extraction from camel urine against Mycobacterium tuberculosis bacteria (MTB) that causes Tuberculosis (TB) disease and determine the period of treatment required for effectiveness comparing with Rifampicin (RIF) antibiotic. This study depended on an experimental design that used 10 random samples of MTB from King Fahad Armed Hospital (KFAH) Processed. The study was conducted in two auto-analyzer systems BACTEC 960 culture system and GeneXpert MTB/RIF assay with five different PMF concentrations. In total, all concentrations of PMF drug except $10 \%$ compared with the RIF antibiotic, showed some effectiveness on MTB. In summary, these results showed a non-significant effect of the PMF concentrations except $10 \%$ that indicated as the ideal concentration of this drug that reduces the number of bacteria. Further statistical tests revealed the P-value of the $F$ test was less than $0.05 \%$. Overall, these results indicate no complete effect comparison between growth control of MTB treated with RIF and treated with PMF except $10 \%$ that need further studies.
\end{abstract}

Keywords: Camel Urine, Mycobacterium tuberculosis, PMF, Rifampin, Tuberculosis, Acid-Fast Bacilli.

\section{INTRODUCTION}

Tuberculosis (TB) is an infectious disease that causes many deaths over 200 years ago and has been known since ancient times. TB occupies the list of the diseases that have been studied in medical history. In 2012; the World Health Organization (WHO) reported an annual TB incidence rate in Kingdom of Saudi Arabia (KSA), the third-largest countries in the Middle East, have 18 per 100,000 TB cases in their population [1] Put WHO In KSA, the influx of workers from the various regions of the world is one of the most influential reasons for the spread of the disease, especially from Southeast of Asia and the Indian subcontinent where TB is a significant public health problem. The ministry of health put many of the regulations for these workers to have a medical examination before their entry. Despite its safety and efficacy, poorly ventilated cramped areas increase the number of affected workers in KSA [2].

Camel urine is a liquid natural product, which has been used traditionally in the treatment of many diseases in Arabic countries, especially in Saudi Arabia. PMF (Prophet Medicine fraction), is the biologically active fraction isolated from dried camel urine (PM701) and approved its ability in many previous types of research [3]. Several studies show that camel urine has been work as an antimicrobial agent for many species of bacteria for example Staphylococcus aureus, Pseudomonas aeruginosa and Salmonella spp [4]. Previous research has indicated that camel urine and camel milk indicators have a positive impact on the inhibition of biological effects of mycotoxins produced by Aspergillus flavus and Aspergillus niger after using high concentrations when low concentrations show no significant inhibitory effect on fungal growth [5]. Khorshid's et al. [6] demonstrated that PMF 
works as a selective anticancer agent without any nephrotoxic or hepatotoxic effects which were indicated by the non-significant changes of both kidney and liver functions. PMF has also proven to be a safe fraction even with high doses or long periods of the therapeutic dose [7].

Moreover, it can function as an antimicrobial agent against four fungi species and to some Gram-negative and Gram-positive bacteria [3]. The treatment rate for tuberculosis is about 14\%; resistant bacteria of tuberculosis causes the rest. This rate is due to many reasons such as the length of treatment (between 6 and eight months), the high resistance of the bacteria and therefore the secondary result of this drug [8]. Therefore, this project was conducted to explore the effect of PMF on TB growth. Recent evidence suggests that camel milk and camel urine is a source of nutrients but also as a source of bioactive agents with therapeutic properties [9]. Ths research aimed to test the antibacterial effect of the PMF a fraction from dried camel urine against Mycobacterium tuberculosis and compare with Rifampicin activity the most common antibiotic used to treat these diseases.

\section{MATERIALS AND METHODS}

Sputum has been collected between November 2016 to April 2017 in King Fahad Arm Forces Hospital in Jeddah. The samples were selected based on a degree of growth in BACTEC-960 system and the viability of bacteria, ten samples were in good conditions, and 15 samples were decomposed. The patients were labelled with the number of hospital code, and the control group is labelled with the actual name of the antibiotic.

\section{Ethical approval}

All work was approved by the Research and Ethics Committee in King Fahad Armed Hospital, Jeddah, Saudi Arabia, and the University of King Abdul-Aziz, Faculty of Science, Biology department.

\section{Laboratory procedures: \\ Identification of Mycobacteria by Smear Examination:}

Identification smear microscope is a follow-up step recommended by WHO to identify the Mycobacterium species. ZiehlNielsen Staining is a technique called Acid-Fast bacilli smear (AFB) which used heat-fixed slides, stained with carbol fuchsin using a hot Ziehl-Neelsen stain and finally examined under the light microscope using a 100X magnification [10].

\section{The effectiveness of PMF against MTB \\ The solvent of PMF}

A water suspension of PMF extraction was found by solvent dried camel urine (PM701) in methyl alcohol and prepared diffident consecrations [11].

\section{BACTEC MGIT 960 system:}

The susceptibility test was performed for ten samples to measure the PMF effectiveness and gold line of antibiotics against MTB in BSCTEC system 960. All isolates were used for Susceptibility testing by using final drug concentrations as shown in Fig. 1. The identification of resistant MTB isolates from these specimens by susceptibility test was for first line antibiotic-MTB drugs, the rifampicin (RIF) isoniazid (INH), streptomycin (STR), and ethambutol (EMB) which were appraise using BACTEC MGIT 960 SIRE Kit [12] and compared with other group of tubes for PMF concentrations doses that including 5, 7.5, 10, 12.5 and 15\% respectively. All isolates of MTB that showed growth on cultures was saved at -80C deep freeze for further molecular investigations. 


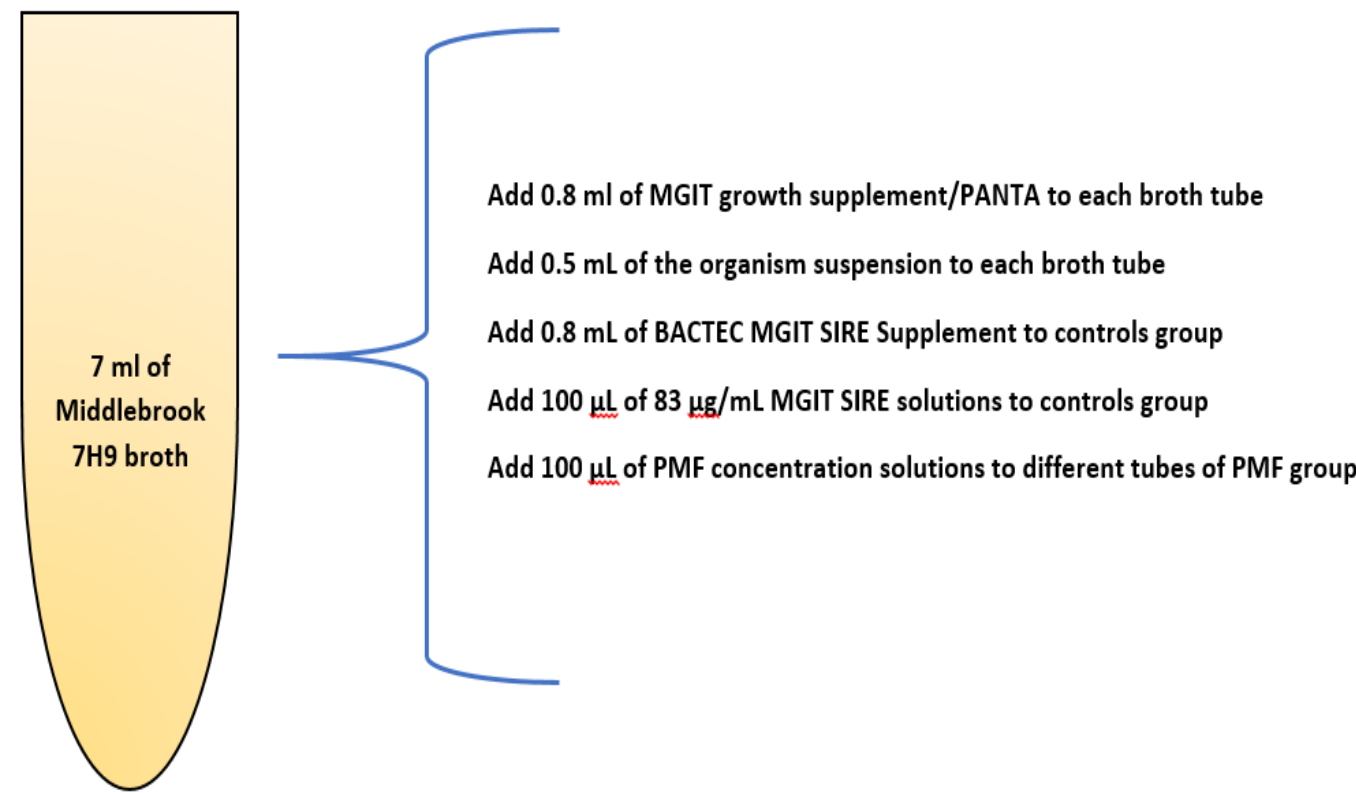

Fig. 1: the direction of preparation the test tubes of Susceptibility Testing

\section{Gene Xpert MTB/RIF assay}

The GeneXpert MTB/RIF Real time-PCR assay kit was performed depending on instructions inside the manufacturer's package of the test [13]. All specimens that were cultured in MIGT (Mycobacteria Growth Indicator Tube) tubes run in GeneXpert; they give the same results of the number of bacteria as in BACTEC 960 system. The results indicate the presence or absence of MTB bacteria and RIF gene resistance, estimated by concentration high, medium, low, and very low indication curves. MTB is known once a 2 of the five $r p o B$ - probes indicate with a positive signal with cycle threshold (CT) values that are $\leq 38$ and that different by not more than two cycles.

\section{Confirmation test}

Contamination test (Purity checks)

The analysis was checked once at the beginning of the optimization check and was therefore checked once again at the top of the analysis by a confirmation test. The simplest technique for this study was found to be the use of incubation broths in sheep agar to prevent contamination of the MGIT broths used in the assay [14]. Medium broths were trailed on sheep agar and incubated for 24 hours in an incubation machine. The results indicate the presence or absence of spices of the bacteria.

\section{Check the properties of PMF on a culture medium}

However, a second check was determined to verify the PMF viability work based on the PMF activity. The culture medium was ready with PMF, inoculated with MTB and $E$. coli for twenty-four hours. The lack of MTB results and therefore the presence of $E$. Coli attributable to the middle property for E. coli.

\section{Statistical analysis}

Data was collected by the BACTEC system and GeneXpert reports, print out from this instrument, and the results were formatted in an excel sheet and were analyzed using the SPSS (Statistical Package for Social Sciences, USA). The significant results were recorded when the $\mathrm{p}$-value is $<0.05$. The $\mathrm{p}$-value was applied to compare the number of MTB to the effect of two drugs PMF with different concentrations and RIF by using two systematic way BACTEC 960 and GeneXpert MTB/RIF.

\section{RESULTS}

The results obtained from the BACTEC system analysis of MTB in PMF group is shown in Fig. 2. Results of this test showed high (i.e. more than 10000 growth unit) mean of 104 isolated samples. However, $12.5 \%$ of MTB cells with PMF did not increase. The following samples show the addition of MTB, a significant increase in the PMF was recorded as well. The most surprising aspect of the data is in the $10 \%$ PMF; a positive correlation between MTB and PMF. Results of this concentration indicated the mean of MTB were minimum less than 2500 growth unit compared with other concentrations. The significant main effects of the PMF influencing the numbers of bacteria in 10\% concentration. Where the graph shows in Fig. 2 that the lowest numbers of bacteria were at $10 \%$ concentration, in all ten samples. 


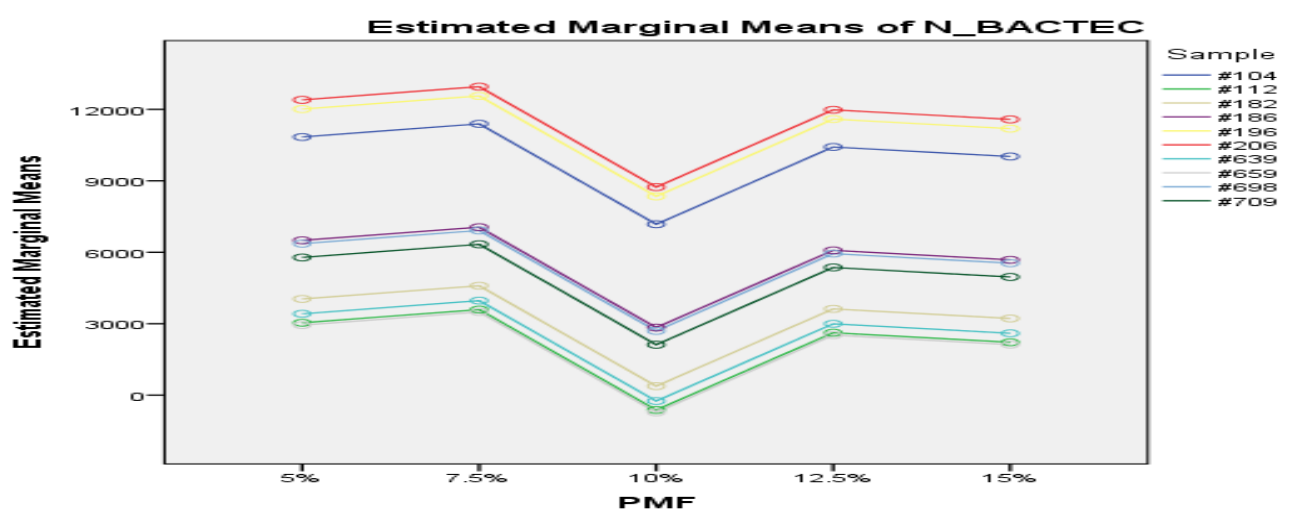

Fig 2. Show the direction of the mean numbers of bacteria.

The correlation between PMF and Rifampicin antibiotic was tested in GeneXpert assay and presented in Table 1. Results of this correlation between two drugs were showed a medium level of MTB in all samples compared growth control and Rifampicin antibiotic against different PMF concentrations.

Figure 3 shows the direction of the mean of $\mathrm{Ct}$ that used to detect the main effects of five rpoB-probes that are complementary to 81-bp RIF resistance-determining region of the wild-type $r p o B$ gene. Where the graph shows, the lowest Ct was at $10 \%$ and Probe A. Where to conclude that the significant effect for each of the PMF concentration, and Probe/SPC on the threshold cycle, as the $P$-value of the F test less than $0.05 \%$.

Table 1. Presents the results obtained from the RT-PCR analysis of the GeneXpert system

\begin{tabular}{|c|c|c|}
\hline \multirow{4}{*}{10} & Controls & \multirow{2}{*}{ Level of MTB } \\
\cline { 2 - 3 } 10 & PMF Concentrations & \\
\cline { 2 - 3 } MTB Samples & Growth factor & High \\
\cline { 2 - 3 } & Rifampicin & Low \\
\cline { 2 - 3 } & $5 \%$ & Medium \\
\cline { 2 - 3 } & $7.5 \%$ & Medium \\
\cline { 2 - 3 } & $10 \%$ & Medium \\
\cline { 2 - 3 } & $12.5 \%$ & Medium \\
\hline
\end{tabular}

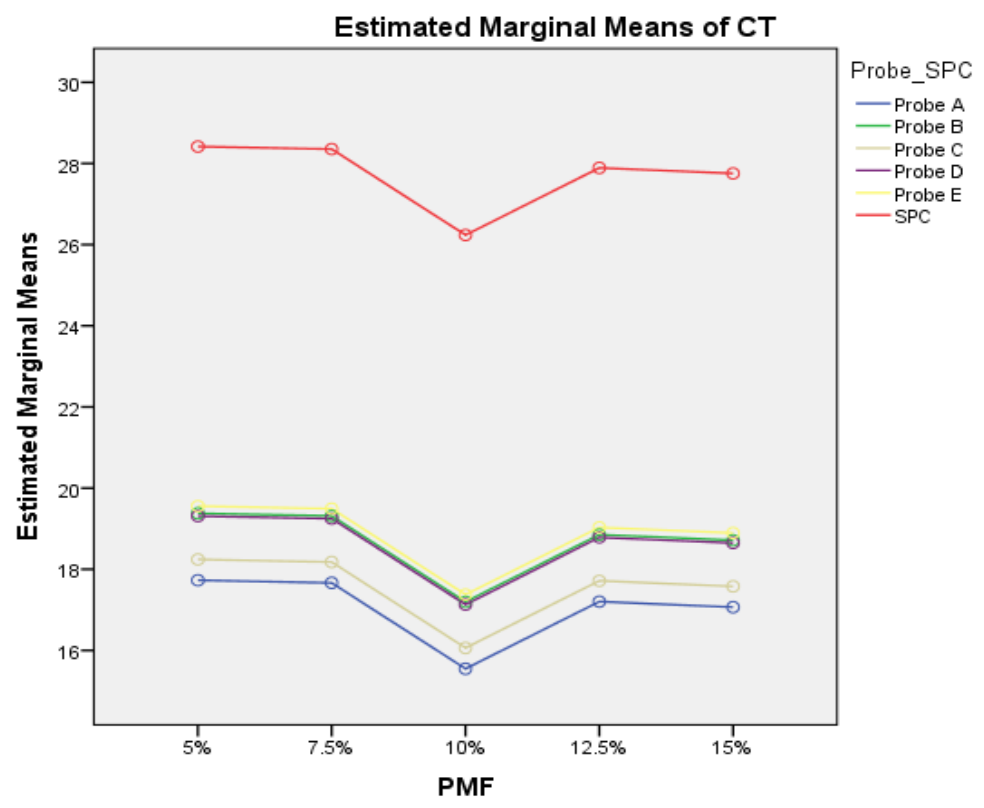

Fig 3. Show the direction of the mean $\mathrm{Ct}$. 
Discordance examined across all the methods and with all samples used in this auto-analyzer. Most isolates completely concordant results across two methods; however, sample No. 206 shows difference reaction with GeneXpert against BACTECT 960 to susceptibility test for gold line antibiotics used in both assays. The reaction depends on the unique structure of MTB isolate from this patient. The final set of analyses examined by Check properties of PMF on Nutrient agar provides as shown in Fig. 4 (MBT don't grow on N. agar exclusively with or without treatment??), streaking showed no difference was observed in MTB plats, but there is a positive plat-streaking with $\boldsymbol{E}$. coli bacteria isolated.

\section{DISCUSSION}

Tuberculosis is usually diagnosed when symptomatic individuals seek care at healthcare facilities; incomplete adherence to TB treatment will increase the chance of delayed culture conversion with the continued transmission within the community, al as treatment failure, relapse, and development or amplification of drug resistance RIF. According to the most recent WHO report [8] the threat that TB presents to world health has been considerably heightened by the evolution and unfolding of drug-resistant TB: in 2015, a staggering 480,000 persons across the globe developed multi-drug resistant (MDR)-TB, outlined as TB that's proof against Rifampicin (RIF) and INH (INH), with or while not resistance to different first-line anti-tubercular medicine. Sadly, this example must continue to worsen victimization the XDR-TB to kinds of the un wellness that are functionally untreatable with existing antibiotics [15]. Camel urine has been consumed extensively for years in Saudi Arabia because it believed to be able to treat a large variety of diseases like cold, fever, or even cancer. PM 701 is a yellowish powdered form of camel urine that proved to work as anti-cancer [16]. A water suspension of PM701 was given with different solvents, such as CHCL3, ethyl acetate, n- butanol or extracted with methyl alcohol. The best solvent for extraction was found methyl alcohol and coded fraction PMF [17] that which is our project depends on studying the effectiveness of PMF as an antibiotic agent.

In this research, it was predicted that the PMF substrate would work like an antibiotic, which has many advantages such as; non-toxic and easier to found in Arab areas. The results show an effect comparison between growth control of MTB treated with RIF and MTB treated with PMF doses in 10\%. Our finding differs from prior reviews. The data showed that PMF worked effectively as an antibiotic against dermatophyte fungi (Aspergillus fumigatus, Epidermophyton floccosum, Microsporum Canis, and Trichophyton rubrum), two Gram-positive bacteria (Staphylococcus citrus and Bacillus subtilis) and two Gram-negative bacteria (Escherichia coli and Salmonella typhi). The PMF showed higher antimicrobial activity up to $32 \mathrm{~mm}$ inhibition zone with $\mathrm{MIC}<100 \mu \mathrm{g} / \mathrm{ml}$ [3]. However, the findings of the current study do not support the previous research. Although these results differ from some published study in 2015, Al-Awadi and Al-Judaibi 18] have tested the fact that the same nail ungulate pee can be an extremely effective and resistant antimycotic for the treatment of diseases of human and plant flora, even at higher temperatures, or parallel to the discoveries. This is also consistent with our previous observations, which showed that uniform nail ungulate operated at low concentrations for the growth of rental flora, while inhibition recorded once high levels of victimization [5].

Important findings in our analysis are that the PMF acts at $10 \%$ concentration, showed effective work compared with controls. the present results, the previous study in 2005 [19] showed that PM 701 (Dried camel urine) works effectively as anti-cancer with cancer cells (Lung cancer cells A549) in low dose (2ml substrate: 100,000 ml media). This study produced results which corroborate the findings of a great deal of the previous work in this field that PMF indeed at low concentrations [19]. These results understand with caution. Future studies on the current surface unit suggested.

This study was aimed at evaluating the performance of PMF antibiotic agent against MTB. In this study, the sample size was 10, that was should be 30 samples or more which could be used for statistical analysis. However, because of the lack of financial resources and enough samples are not available in the laboratory, this gap exists but was replaced by the using different concentrations of PMF to treat bacteria with up to 3 times trials. This led to giving weight to the results produced.

\section{CONCLUSION}

Our data suggest that MTB is not affected by PMF at a concentration 5-15\% whereas10\%of PMF give promising results compared with control, as evidenced by our results on ten samples. However, further research needs to be conducted on a wider scale to confirm our preliminary data. Overall, these results indicate that no complete effect comparison between growth control of MTB treated with RIF and treated with PMF except $10 \%$ that need further studies.

\section{CONFLICT OF INTEREST}

The authors declare no conflicts of interest.

\section{ACKNOWLEDGEMENT}

This research was supported by Tuberculosis patients in King Fahad Armed Hospital. We thank our colleagues from PCR department who provided insight and expertise that greatly assisted the research. We thank Turki Al - Adwani, Laboratory technician for assistance with BACTEC MGIT 960 system method, and Faten Khorshid, Professor, King Abdulaziz University for comments that greatly improved the manuscript. 


\section{REFERENCES}

1. Al-Hajoj, S. and B. Varghese, 2015. Tuberculosis in Saudi Arabia: the journey across time. The Journal of Infection in Developing Countries, 9 (03): 222-231.

2. Méda, Z. C., I. Sombié, O. W.C. Sanon, D. Maré, D. E. Morisky and Yi-M. A. Chen, 2013. Risk Factors of Tuberculosis Infection Among HIV/AIDS Patients in Burkina Faso, Aids Research and Human Retroviruses, 29:7, 1045-1055.

3. El-Shahawy A, El-Sawi N., Backer W.S., Khorshid F.A., and Geweely N.S. Spectral 2010. Analysis, Molecular Orbital Calculations and Antimicrobial Activity of PMF-G Fraction Extracted From PM-701. Int. J. of Pharma and Bioscience, $1(2): 1-19$.

4. Ahmed, M.E., A. Abdalla and H.E. Ahmed, 2008. Bacteria Associated with Healthy Sudanese Camel Urine and Susceptibility of Some Bacteria of Human Origin to Camel Urine. Sudan J. Vet. Res., 23: 79-82.

5. Al-Abdalall, A.H.A., 2010. The inhibitory effect of camel's urine on mycotoxins and fungal growth. African Journal of Agricultural Research, 5(11): 1331-1337.

6. Khorshid F.A., H., Shazly, A., Al-Jefery and A.A. Osman 2010. Dose Escalation Phase I Study in Healthy Volunteers to Evaluate the Safety of a natural product PM 701. Int. J. of pharmacology and toxicology, 5(3): 91-9.

7. Khorshid, F., S. Rabah, H.A. Abuaraki, A. Ali, S. Noor and H. Alkabkaby, 2015. Safety of Oral Administration of PMF a Fraction Derived from Camel Urine: Acute Study on Mice. Nternational Journal of Emerging Technology and Advanced Engineering, 5: 365-370.

8. World Health Organization 2018. Global tuberculosis report, HO/CDS/TB/2018.20: 1-277.

9. Gader, A.G.M.A. and A.A. Alhaider, 2016. The unique medicinal properties of camel products: A review of the scientific evidence. Journal of Taibah University Medical Sciences, 11(2): 98-103.

10. Madison, B., 2001. Application of stains in clinical microbiology. Biotechnic \& Histochemistry, 76(3): 119-125.

11. Khorshid, F., 2009. The effect of lyophilized PM701 and its fractions on A549 cancer cells. Trends Med Res, 4 (1): 8-15.

12. Siddiqi, S.H. and S. Rüsch-Gerdes, 2006. The MGIT TM Procedure Manual. Retrieved from, 1-89.

13. Raviglione, M., 2010. Cephei the Xpert MTB/RIF Assay. Retrieved from, 1-4.

14. Scarparo, C., P. Ricordi, G. Ruggiero, and P. Piccoli, 2004. Evaluation of the fully automated BACTEC MGIT 960 system for testing susceptibility of Mycobacterium tuberculosis to pyrazinamide, streptomycin, isoniazid, rifampin, and ethambutol and comparison with the radiometric BACTEC 460TB method. Journal of clinical microbiology, 42(3): 1109-1114.

15. Reiche, M.A., D.F. Warner and V. Mizrahi, 2017. Targeting DNA replication and repair for the development of novel therapeutics against tuberculosis. Frontiers in molecular biosciences, 4: 75.

16. Alghamdi, Z. and F. Khorshid, 2012. Cytotoxicity of the urine of different camel breeds on the proliferation of lung cancer cells, A549. J Nat Sci Res, 2(5): 9-16.

17. Khorshid, F. A., A.M. Osman and E. Abdel-Sattar, 2009. Cytotoxic Activity of Bioactive Fractions from PM 701. Electronic Journal of Environmental, Agricultural \& Food Chemistry, 8(12).

18. Al-Awadi, A. and A. Al-Judaibi, 2014. Effects of Heating and Storage on the Antifungal Activity of Camel Urine. Clinical Microbiology, 3(6) 1-6.

19. Khorshid, F.A., S.S. Mushref and N.T. Heffny, 2005. An ideal selective anti-cancer agent in vitro I-tissue culture study of human lung cancer cells A549. JKAU-Medical Sciences, 12: 3-19. 